\title{
A DIFFUSIVE SEIR MODEL FOR COMMUNITY TRANSMISSION OF COVID-19 EPIDEMICS: APPLICATION TO BRAZIL
}

\author{
WILLIAM E. FITZGIBBON, JEFFREY J. MORGAN, GLENN F. WEBB, AND YIXIANG WU
}

\begin{abstract}
A mathematical model incorporating diffusion is developed to describe the spatial spread of COVID-19 epidemics in geographical regions. The dynamics of the spatial spread are based on community transmission of the virus. The model is applied to the outbreak of the COVID-19 epidemic in Brazil.
\end{abstract}

\section{INTRODUCTION}

Coronavirus disease 2019 (COVID-19) firstly identified in December 2019 has infected over 20 million people worldwide as of September 2021 [11, 26]. This disease is caused by a novel coronavirus named SARS-CoV-2. Major epidemics caused by viruses in the family of coronavirus include the SARS-CoV epidemic in 2003 and the MERS-CoV epidemic in 2012. A distinguished feature of COVID-19 is that a large proportion of people infected with this disease do not show symptoms [54], and the symptoms of the patients vary from mild symptoms to severe illness [26]. Moreover, infected people who do not show symptoms can also transmit the disease, and these features make the disease difficult to control 22.

Mathematical treatments of the spatial spread of COVID-19 have used probabilistic methods [10, 17, neural networks methods [27, 38, data analysis methods [5, 12, 33, 21], and differential equations methods 4, 17, 14, 15, 20, 40, 41, 44, 47. Reaction-diffusion equations have been used extensively to model the spatial spread of epidemic diseases (see, for example, 1, 2, 6, 13, 19, 18, 29, 32, 37, 49, 50, 53, ). Partial differential equation models for COVID-19 with geographical data have appeared in [3, 24, 48, In particular, the authors in [3] use a kinetic transport model to simulate the outbreak of COVID-19 epidemic in Italy; the authors in 24] use a diffusive compartmental model to simulate the spatial spread of COVID-19 in three cities of Italy, USA, and Brazil.

In this paper, we will use a reaction-diffusion model to simulate the community transmission of COVID-19, which plays a major role in the geographical spread of the epidemics. Understanding the role of community transmission in the geographical evolution of COVID-19 epidemics can contribute to the development of measures for controlling the pandemic. Community transmission cases of COVID19 are distinguished from sporadic cases, clusters of cases, travel-based cases, and country-wide cases 52. Community transmission occurs with large numbers of local transmissions, without direct source of exposure identifications [51. Community transmissions are measured in daily number of confirmed cases per specified number of inhabitants in the designated community. The World Health Organization provides specific recommendations for public health communication and engagement for reduction of COVID-19 community transmission [52]. After the outbreak of COVID-19, many governments have put

Received by the editors 4 August 2021; accepted 5 November 2021; published online 18 November 2021.

2010 Mathematics Subject Classification. Primary 92A15; Secondary 35K57, 35Q92.

Key words and phrases. Reaction-diffusion equations, epidemic models, spatial transmission, COVID-19. 
in place stringent measures including: travel limitation or embargoes, lock downs or partial lock downs, requirements for home work, masking, social distancing, and the frequent disinfecting of commonly used facilities. It is reasonable to assume, when one considers the regional or national spread as opposed to the global or transnational spread, that transmission over short distances is the dominant factor. Therefore, we do not consider the long distance transport of susceptible and infected individuals, while local movement of the virus is indirectly viewed as as the movement of infectious individuals, described with diffusion processes. This motivates us to use a reaction-diffusion model with diffusion terms for infected compartments and without diffusion terms for susceptible and recovered compartments. Our approach has been adopted by recent papers [20, 32]. In particular, in [32], the authors have used such a model to simulate the spatial outbreak of influenza in Puerto Rico.

The organization of this paper is as follows. In Section 2, we develop a mathematical model of COVID-19 community transmission, and state our theoretical results. In Section 3, we provide numerical simulations of our model to the spatial spread during the outbreak stage of the COVID-19 epidemic in Brazil. In Section 4, we draw a conclusion. The proofs of the mathematical results are presented in the appendix.

\section{The Model}

We assume that the host population is confined to a bounded region $\Omega \subseteq \mathbb{R}^{N}$ with smooth boundary $\partial \Omega$ that lies locally on one side of the boundary. The spatial density of the susceptible class at location $x \in \Omega$ and time $t \geq 0$ is represented by the variable $S(x, t)$. The asymptomatic infected class is represented by $E(x, t)$, while the infected and recovered classes are represented by the variables $I(x, t)$ and $R(x, t)$, respectively. Individuals are recruited to the exposed class from the susceptible class via contact with either the asymptomatic infected class or the symptomatic class. We model this process with standard incidence mechanisms

$$
f(x, S, E, I, R)=\sigma_{1}(x) \frac{S E}{S+E+I+R}+\sigma_{2}(x) \frac{S I}{S+E+I+R},
$$

where $\sigma_{1}(x)$ and $\sigma_{2}(x)$ are transmission coefficients. We assume that asymptomatic infected individuals leave the class at a rate constant $\lambda$, which means that individuals spend an average time $1 / \lambda$ in this class. For simplicity, we assume that all the individuals leaving the asymptomatic infected class will join the symptomatic infected class, although many COVID-19 patients have mild or no symptoms. We assume that the infected individuals recover or die at a constant rate $\gamma$, which means that infected individuals spend an average time $1 / \gamma$ in this class (if an infected individual does not show any symptoms before recovery, then that individual spends zero time in this class). Our model differs from the standard diffusive SEIR model by virtue of the fact that the exposed class $E$ is also infectious. This represents the asymptomatic phase of COVID-19.

The forgoing considerations lead to a degenerate reaction-diffusion system of the form

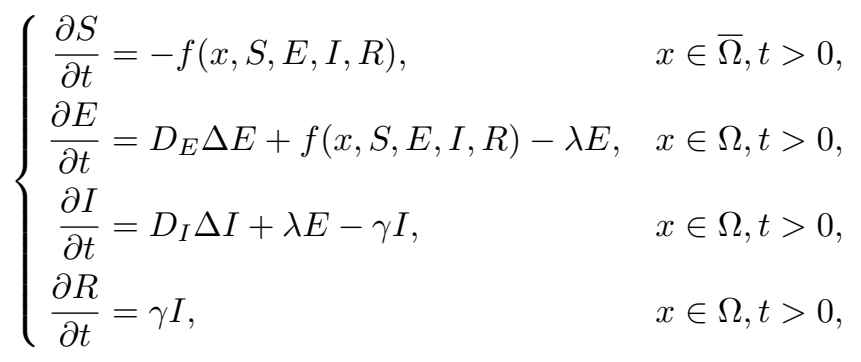


where $D_{E}$ and $D_{I}$ are positive diffusion coefficients. On $\partial \Omega$, we impose homogeneous Neumann boundary conditions:

$$
\frac{\partial E}{\partial \eta}=\frac{\partial I}{\partial \eta}=0, \quad x \in \partial \Omega, t>0,
$$

which means that the individuals cannot cross the boundary $\partial \Omega$. Finally, we impose initial conditions:

$$
\left\{\begin{array}{l}
S(x, 0)=S_{0}(x), \quad x \in \bar{\Omega}, \\
E(x, 0)=E_{0}(x), \quad x \in \Omega, \\
I(x, 0)=I_{0}(x), \quad x \in \Omega, \\
R(x, 0)=R_{0}(x), \quad x \in \bar{\Omega} .
\end{array}\right.
$$

To include mass action infection mechanism $f(x, S, E, I, R)=\sigma_{1}(x) S E+\sigma_{2} S I$ into our model, we make the following assumptions:

(A1) $f: \bar{\Omega} \times \mathbb{R}^{4} \rightarrow \mathbb{R}$ is continuously differentiable;

(A2) $f(x, S, E, I, R) \geq 0$ for $(x, S, E, I, R) \in \bar{\Omega} \times \mathbb{R}_{+}^{4}$;

(A3) $f(x, 0, E, I, R)=0$ for $(x, E, I, R) \in \bar{\Omega} \times \mathbb{R}_{+}^{3}$;

(A4) There exists a continuously differentiable function $g: \mathbb{R}_{+} \rightarrow \mathbb{R}_{+}$and a polynomial function $P: \mathbb{R}_{+}^{2} \rightarrow \mathbb{R}$ such that $g(0)=P(0,0)=0$ and $f(x, S, E, I, R) \leq g(S) P(E, I)$ for $(x, S, E, I, R) \in \bar{\Omega} \times \mathbb{R}_{+}^{4}$.

We have the following result about existence of nonnegative solutions of the model:

Theorem 2.1. Suppose that (A1)-(A4) hold and there exists $\epsilon \in(0,1)$ such that $S_{0}, E_{0}, I_{0}, R_{0} \in C^{\epsilon}(\bar{\Omega})$ are nonnegative. Then there exists a unique quadruple $(S, E, I, R)$ providing a classical, component-wise nonnegative solution of (2.1)-(2.3) on $\bar{\Omega} \times[0, \infty)$. Moreover, there exists $M>0$ such that

$$
0 \leq S(x, t), E(x, t), I(x, t) \leq M \quad \text { for all } \quad x \in \Omega, t>0 .
$$

We have following result about the asymptotic behavior of solutions of the model:

Theorem 2.2. Suppose that (A1)-(A4) hold and there exists $\epsilon \in(0,1)$ such that $S_{0}, E_{0}, I_{0}, R_{0} \in C^{\epsilon}(\bar{\Omega})$ are nonnegative. Let $(S, E, I, R)$ be the solution of 2.1)-(2.3). Then, we have

$$
\lim _{t \rightarrow \infty} E(x, t)=\lim _{t \rightarrow \infty} I(x, t)=0,
$$

uniformly for $x \in \bar{\Omega}$, and there exists a function $S_{\infty}$ on $\bar{\Omega}$ such that $0 \leq S_{\infty}(x) \leq S_{0}(x)$ and

$$
\lim _{t \rightarrow \infty} S(x, t)=S_{\infty}(x), \quad \text { for each } x \in \bar{\Omega} .
$$

Furthermore, $S_{\infty}>0$ a.e. on any open subset of $\Omega$ on which $S_{0}>0$.

The proofs of these results are similar to those in [20, and we have attached them in the appendix.

\section{Numerical Simulations}

We apply our model to the spatial spread of COVID-19 in Brazil during the early outbreak stage. The outbreak in Brazil began in Sao Paolo in late February and early March, 2020. The first cases arose from international airline travelers to Sao Paolo, primarily from Italy [5]. The epidemic propagated rapidly from the Sao Paolo epi-center to other areas of Brazil. The states of Sao Paulo and Rio de Janeiro both identified community transmission on March 13, 2020 [10]. On March 20 the Brazilian Congress declared a Public Calamity State, on March 19 the Federal District of Brasilia decreed mandatory social restrictions, and on March 23 Sao Paolo State decreed mandatory quarantine measures [46]. Other cities and states soon followed, including Santa Catarina, Bahia, Esprito Santo, and Minas Gerais [42, 45]. 
COVID-19 transmission is dependent on two categories of travel: (1) inter-state travel (long range) and (2) community travel (short range). Inter-state travel between regions of Brazil depends on commercial interactions, transport, social gatherings, and other socio-economic factors. Inter-state mobility between major urban centers in composed primarily of airline travel, and consequently, inter-state COVID-19 spread is primarily by air-travel [10. Community travel is based primarily on daily activities and local public transportation. In our model, the diffusion terms represent the local spread of infectious agents. The parameters of transmission in our model are designed to account for the regional characteristics of community transmission based on this community travel. In our simulations, the initial date is set to be March 17, 2020 when social strict measures were implemented to limit inter-state transmissions.

TABle 1. Parameter values in the simulations

\begin{tabular}{c|c|c}
\hline \hline Parameters & Values & References \\
\hline$\sigma_{1}$ & 0.16 per day & Fitted \\
\hline$\sigma_{2}$ & 0.15 per day & Fitted \\
\hline$\lambda$ & $1 / 7$ per day & 30,31 \\
\hline$\gamma$ & $1 / 7$ per day & {$[30,31$} \\
\hline$D_{E}$ & $2 \mathrm{~km}^{2}$ per day & Assumed \\
\hline$D_{I}$ & $1 \mathrm{~km}^{2}$ per day & Assumed \\
\hline
\end{tabular}

Our simulations are based on model 2.1 -2.3 with standard incidence mechanism, and the parameters used are listed in Table 3 . If $D_{E}=D_{I}=0$, at each local point $x \in \Omega$, the basic reproduction number is

$$
\mathcal{R}_{0}(x)=\frac{\sigma_{1}}{\lambda}+\frac{\sigma_{2}}{\gamma}=2.17
$$

We use the finite element solver in the partial differential equation package of Matlab to solve (2.1)2.3. The boundary $\partial \Omega$ is constructed using the geographic boundary data of Brazil. Figure 1 shows a mesh with 15908 nodes and 7723 triangles for the finite element scheme while more modes and triangles are used in simulations.

At the beginning of the epidemics, we assume that the whole population is susceptible, and therefore the initial value of the susceptible population, $S(x, 0)$, can be described by the distribution of the Brazilian population. The distribution of the Brazilian population data is obtained from [8]. The initial value $S(x, 0)$ is plotted in Figure 2, where we can see that the Brazilian population are mainly concentrated in the Eastern and Southern coast regions.

We obtain the reported COVID-19 case data for each 27 states of Brazil from [23]. We assume that only a fraction, $f=0.2$, of newly infected cases are reported [16, 39]. Therefore, the expression $f \int_{0}^{t} \int_{\Omega_{i}} \lambda I(x, t) d x$ gives the accumulated reported cases of state $i, i=1,2, . ., 27$, at day $t$, where $\Omega_{i} \subset \Omega$ is the domain of state $i$ (we do not take account for the reported cases before $t=0$ for simplicity). The starting point $t=0$ is set to be March 17, 2020 in the simulations. The initial distribution of asymptotically infected people, $E(x, 0)$, of each state is assumed to be proportional to the susceptible population distribution, where the total initial asymptotically infected people in each state is estimated. The total number of initial infected people $\int_{\Omega_{i}} I(x, 0) d x$ for each state $i$ is based on data from [23], while the distribution $I(x, 0)$ is taken to be proportional to the susceptible population distribution.

We solve the model with parameters and initial values described as above. In Figure 3 , we plot the simulated total accumulated COVID-19 cases in Brazil, which match well with the data. We are also 


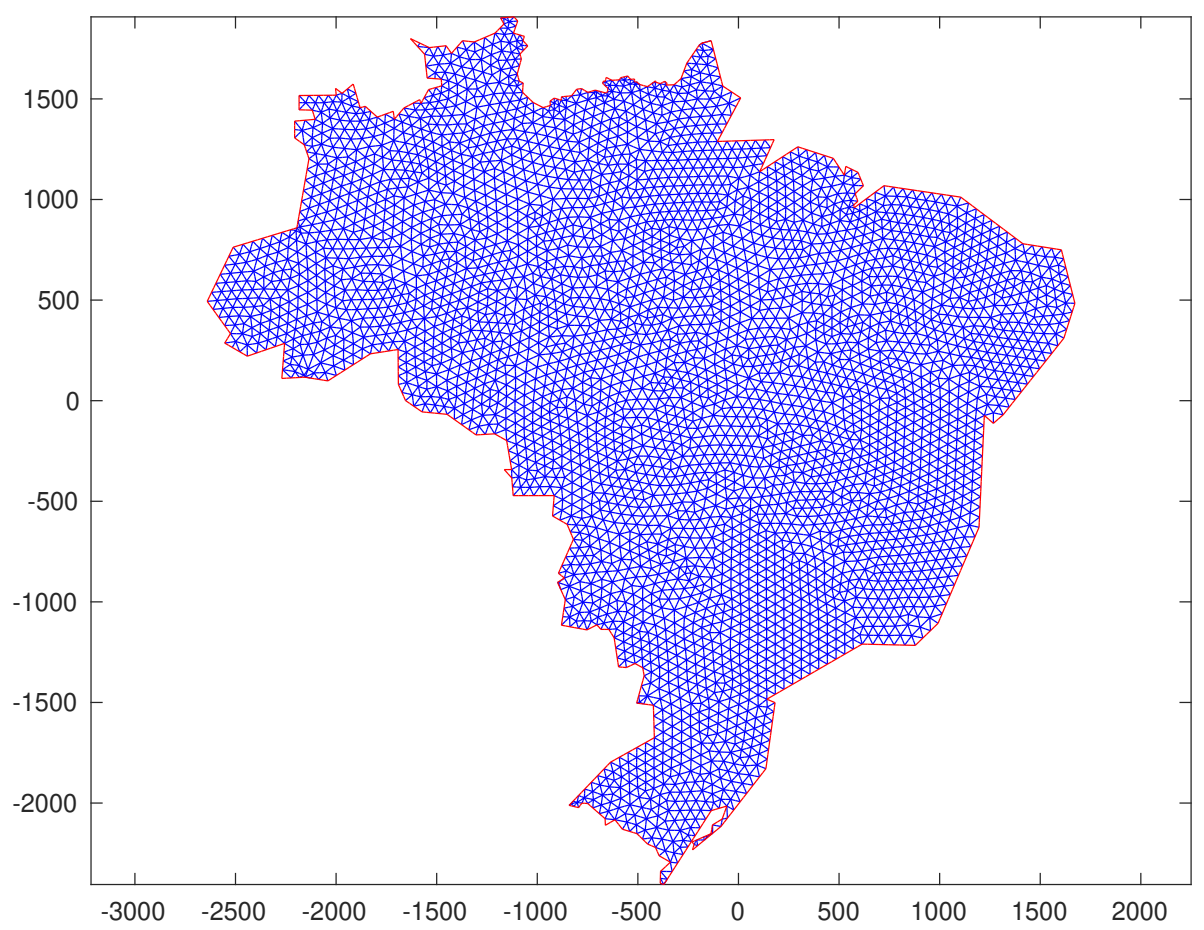

FiguRE 1. Mesh of the model using the geographic boundary of Brazil. This figure shows a total of 15908 nodes and 7723 triangles. In the simulations, we use 376034 nodes and 187049 triangles.

able to compute the accumulated reported cases in each 27 states of Brazil and compare the simulations with data. For most of the 27 states, the simulated accumulated cases match well with the data from 23. In Figure 4, we plot the simulated accumulated reported COVID-19 cases and compare with data for six most populated states: Bahia, Minas Gerais, Parana, Rio de Janeiro, Rio Grande do Sul and Sao Paulo. There are few states that the simulation do not match the data well, which are expected as we assumed uniform in space and time constants for simplicity.

In Figure 5, we graph the simulated distribution of infected COVID-19 cases, $I(x, t)$, in Brazil at March 18 and March 28. From the figure, we can see that the infected cases are concentrated at the most populated areas. To compare with Figure 5, Figure 6 shows the actual infected cases data.

In Figure 7 we plot the simulated number of active cases per 1,000,000 people in each state at different days. In Figure 8, we plot the number of active cases per 1,000,000 people in each state at different days with reported case data from [23]. The simulation values in Figure 7 and the reported data values in Figure 8 are in essential agreement.

Data accessibility: The MATLAB code associated with this article is available at https://github.com/YixiangMath/SpatialSimulations.git 


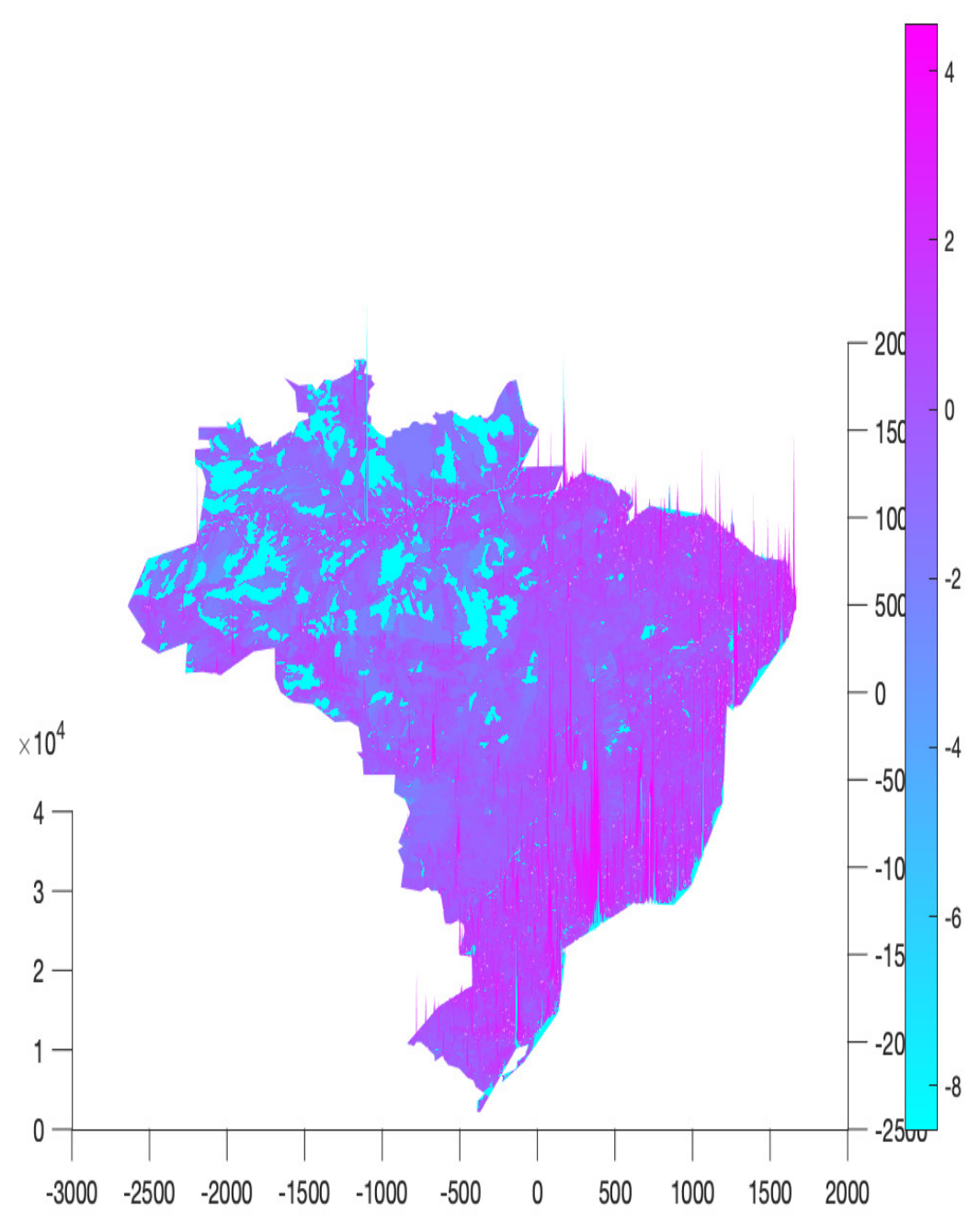

Figure 2. Distribution of Brazilian population, which corresponds to the initial value of susceptible population, $S(x, 0)$, in the simulations. Data source [8]. The $z$-axis of the graph is population density (unit is number of people per $\mathrm{km}^{2}$ ). The figure is colored by taking the $\log$ of the population density, i.e. $\log (S(x, 0))$. 
W. E. FITZGIBBON, J. J. MORGAN, G. F. WEBB, AND Y. WU

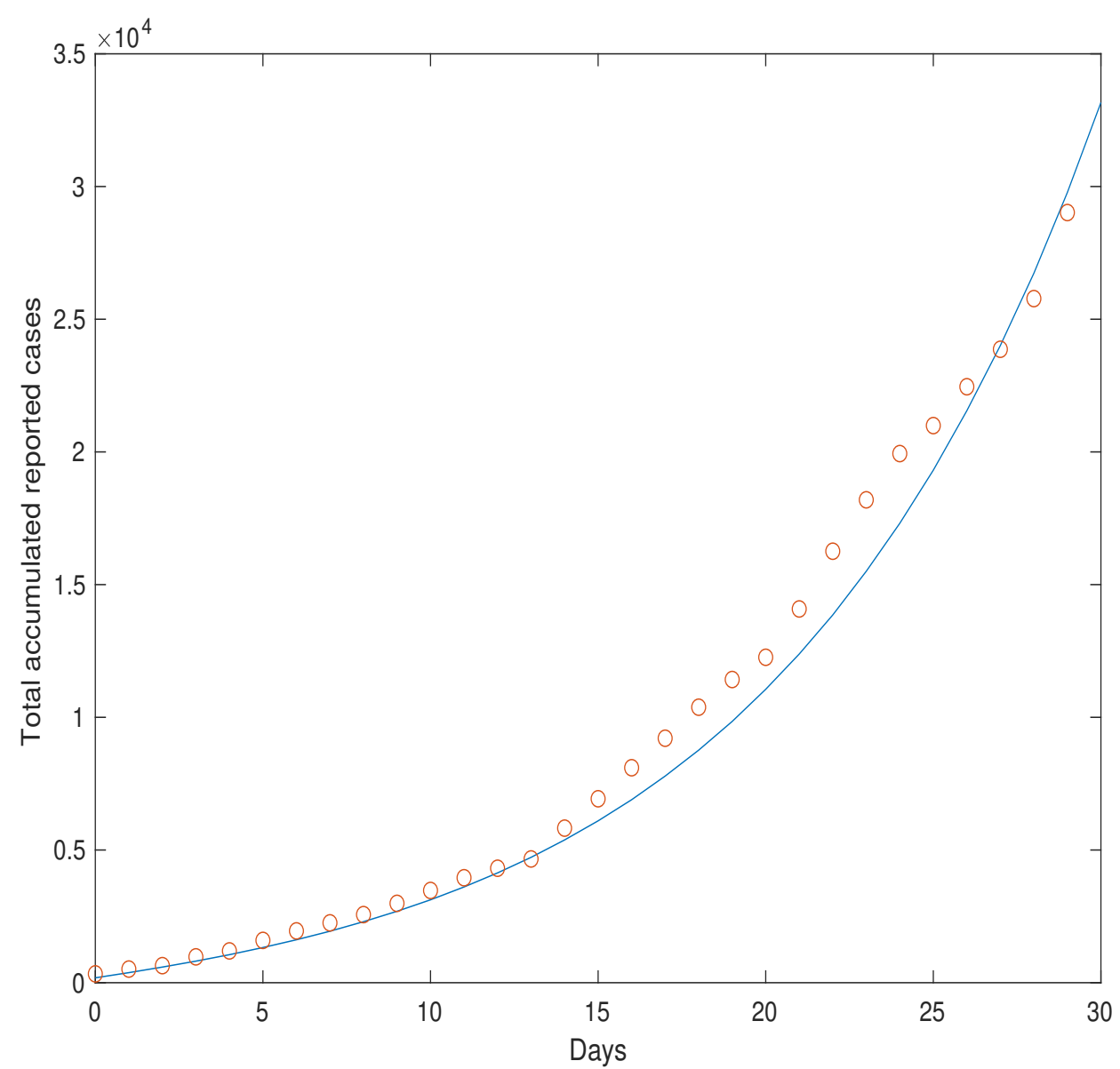

Figure 3. Simulation of accumulated reported COVID-19 cases in Brazil. The initial date is March 17, 2020. The dots correspond to the real data and the curve is from simulation. 


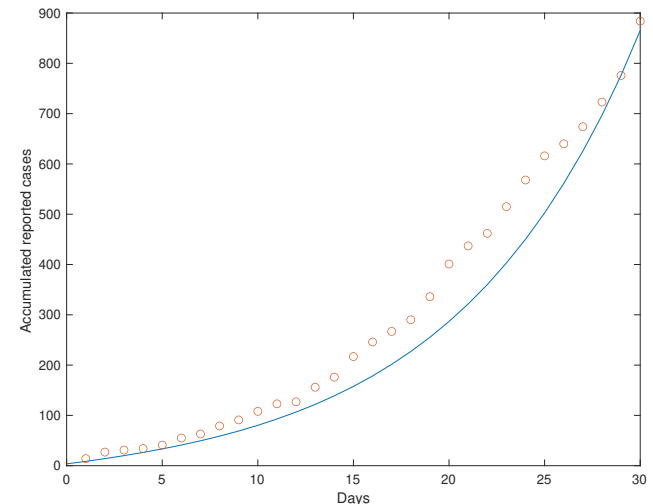

Bahia

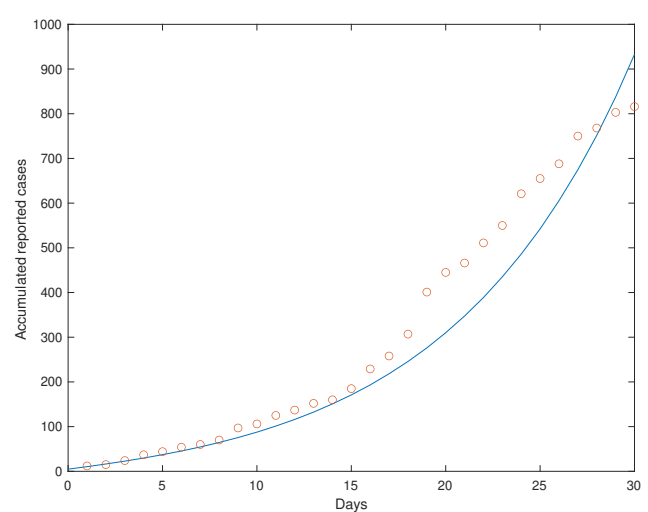

Parana

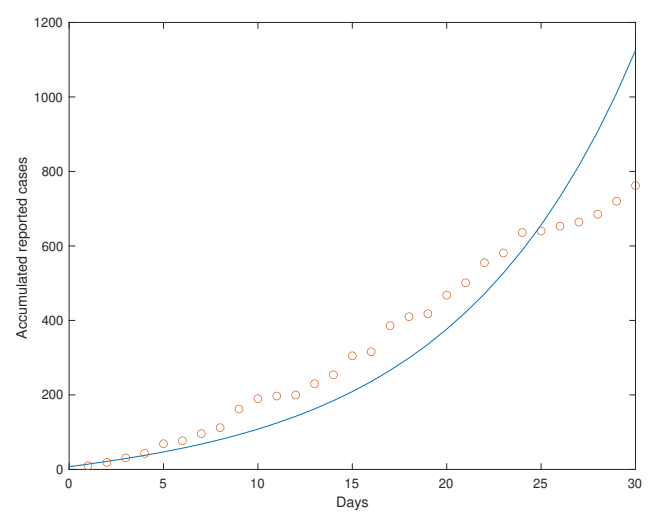

Rio Grande do Sul

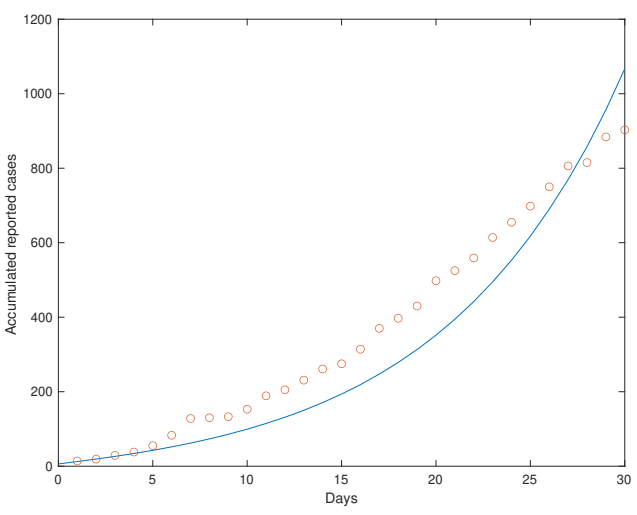

Minas Gerais

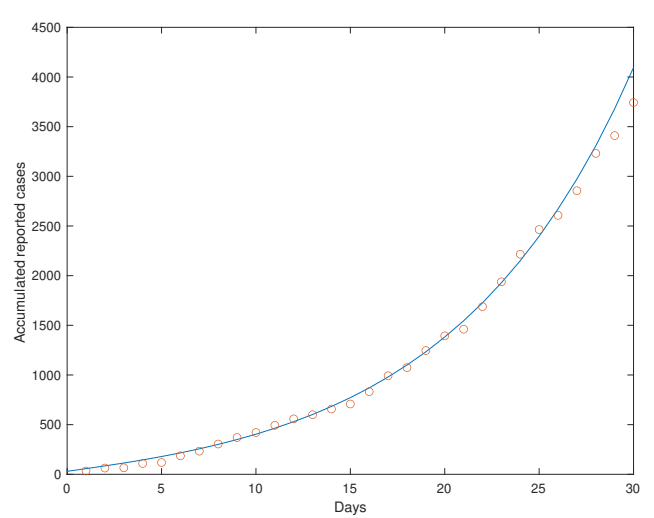

Rio de Janeiro

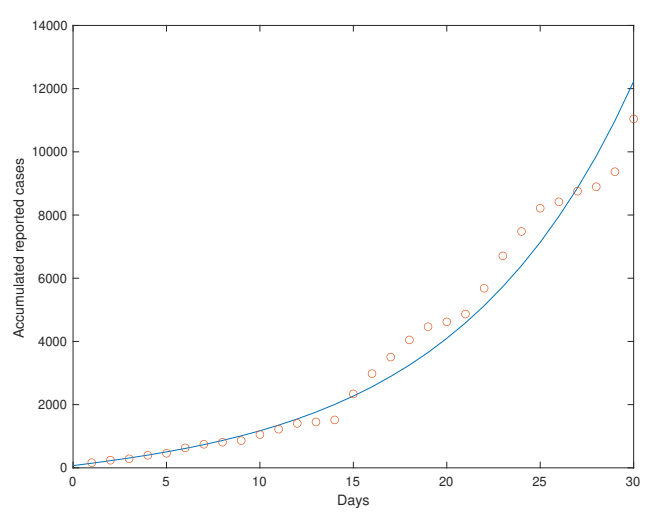

Sao Paulo

FIGURE 4. Simulation of accumulated reported COVID-19 cases for six most populated states of Brazil. The curves are the simulated accumulated cases and the dots are real data. The initial date is March 17, 2020. 

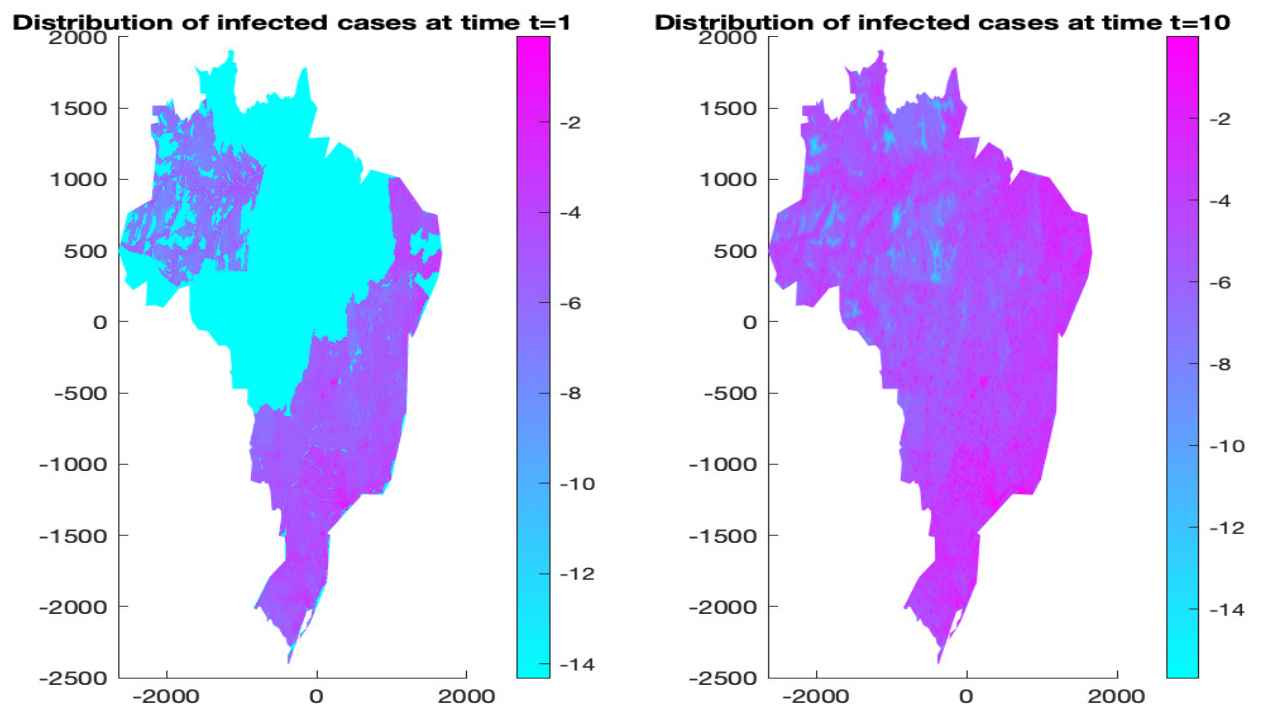

Figure 5. Simulation of the distribution of active COVID-19 cases, $I(x, t)$, in Brazil at March 18 and March 28. The graph is colored by $\log (I(x, t))$.

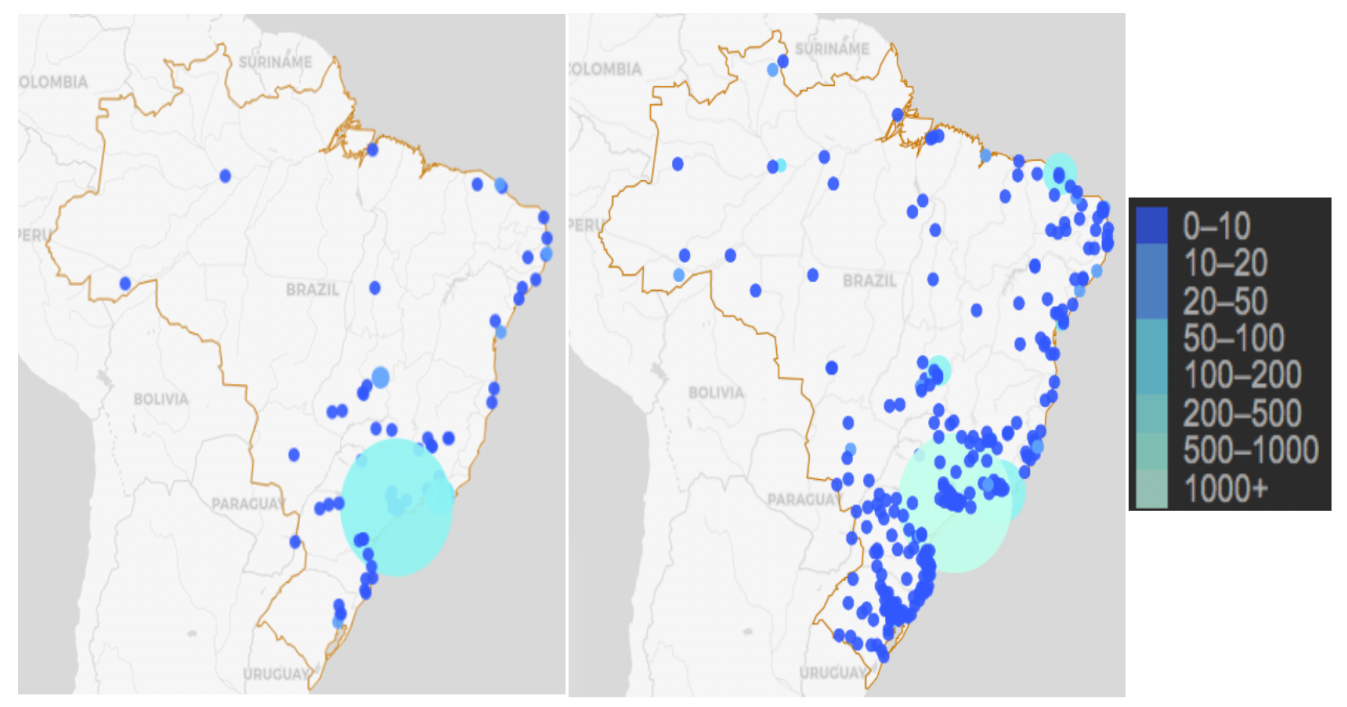

Figure 6. Distribution of active COVID-19 cases in Brazil at March 18 and March 28. This figure is obtained from 23. 

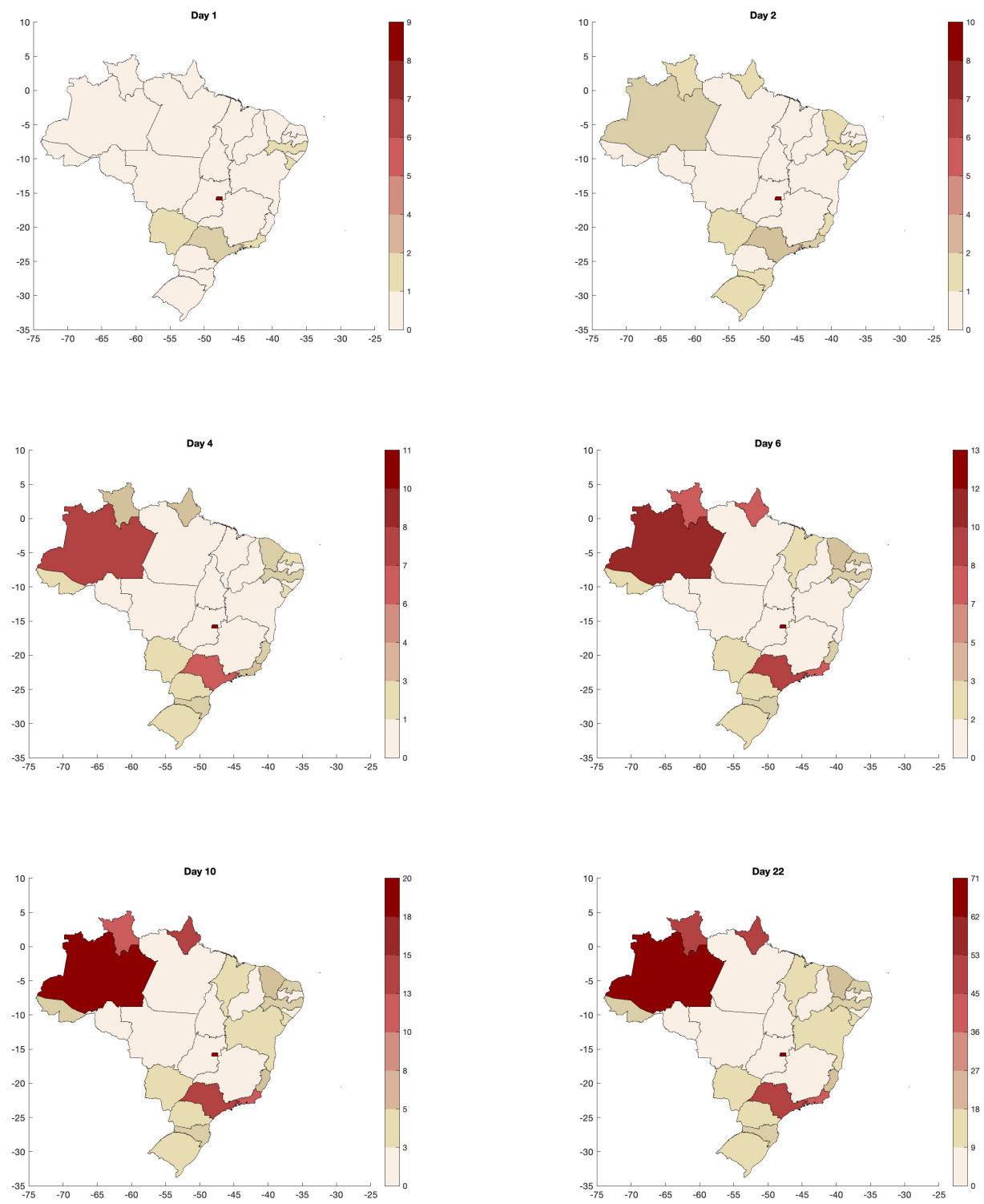

FiguRE 7. Simulation of number of active cases per 1,000,000 people. The initial date is March 17, 2020. 

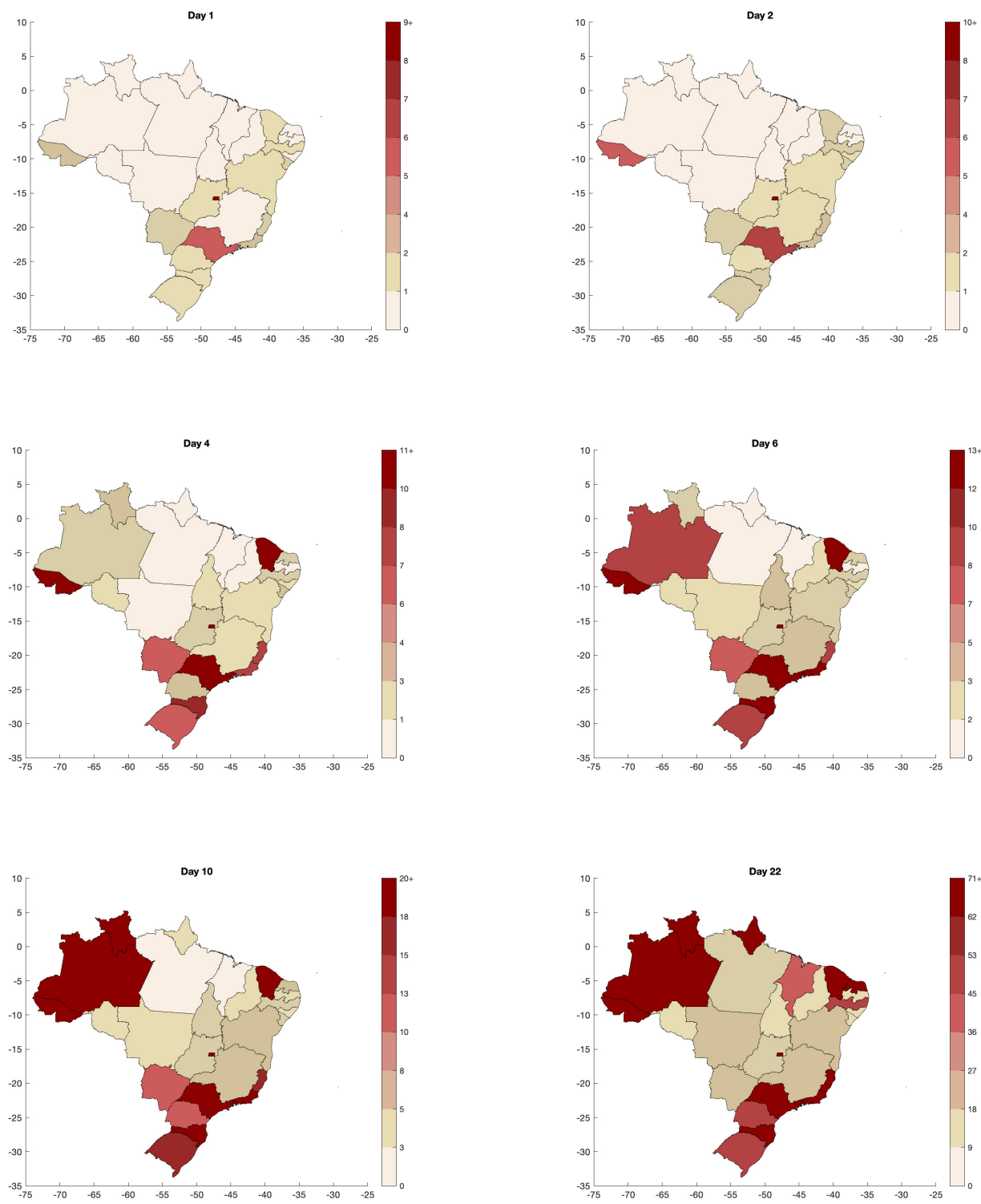

FIGURE 8. Number of active cases per 1,000,000 people (data from [23]). The initial date is March 17, 2020. 


\section{Conclusion}

In this paper, we propose and analyze a diffusive epidemic model for the community transmission of COVID-19. The major feature of the model is that there is no diffusion term for the equation of susceptible compartment, while diffusion term is used to describe the community spread of the virus in the equations of asymptomatic infected and infected compartments. The model has been used to simulate the outbreak of COVID-19 in Brazil. In the simulation, the geographical boundary data of Brazil is used to construct the domain of the model, and the population data of Brazil is used to set up the initial conditions of the model. We use the partial differential equation toolbox in MATLAB to solve the model and use the mapping toolbox to plot the solutions. Using the geographical boundary data of the states of Brazil, we are able to determine and plot infected cases for different states and compare with real data.

Our simulations are also limited. We use spatial uniform parameters in the simulations, and the spatial heterogeneity is reflected mainly in the distribution of the susceptible population. It is desirable to come up with methods to design or estimate spatially heterogeneous parameters for the model. Though the simulated total cases fit the data well at the first thirty days, the simulation will overestimate the epidemic size in the following days. Factors such as quarantine measures, social distancing and masking will affect the transmission of the disease, so it is unlikely to use a model with time-independent parameters to fit the epidemic data over a large time interval.

\section{Appendix}

5.1. Preliminary. For $0 \leq \tau<T<\infty$, let $Q_{\tau, T}=\Omega \times(\tau, T)$. We denote by $L^{p}\left(Q_{\tau, T}\right), 1<p<\infty$, the space of measurable function $\phi: Q_{\tau, T} \mapsto \mathbb{R}$ with the norm:

$$
\|\phi\|_{p, \tau, T}=\left(\int_{Q_{\tau, T}}|\phi(x, t)|^{p} d x d t\right)^{1 / p} .
$$

Given $\theta \in L^{p}\left(Q_{\tau, T}\right)$, let $\phi$ be the solution of the following problem

$$
\begin{cases}\partial_{t} \phi=-d \Delta \phi-\theta, & x \in \Omega, t \in(\tau, T), \\ \partial_{\nu} \phi=0, & x \in \partial \Omega, t \in(\tau, T), \\ \phi=0, & x \in \Omega, t=T .\end{cases}
$$

Note that if $\tilde{\phi}=\phi(x, T-t)$ and $\tilde{\theta}=\theta(x, T-t)$ for $(x, t) \in \bar{\Omega} \times(0, T-\tau)$, then $\tilde{\phi}$ satisfies the following:

$$
\begin{cases}\partial_{t} \tilde{\phi}=d \Delta \tilde{\phi}+\tilde{\theta}, & x \in \Omega, t \in(0, T-\tau), \\ \partial_{\nu} \tilde{\phi}=0, & x \in \partial \Omega, t \in(0, T-\tau), \\ \tilde{\phi}=0, & x \in \Omega, t=0 .\end{cases}
$$

The following lemma comes from [28].

Lemma 5.1. Let $p \in(1, \infty)$ and $0 \leq \tau<T<\infty$. Given $\theta \in L^{p}\left(Q_{\tau, T}\right)$, let $\phi$ be the solution of 5.1. Then, there exists constant $C(p, T-\tau)$ independent of $\theta$ such that

$$
\|\phi(\cdot, \tau)\|_{L^{p}(\Omega)},\|\phi\|_{p, \tau, T},\|\Delta \phi\|_{p, \tau, T} \leq C(p, T-\tau)\|\theta\|_{p, \tau, T}
$$

We will need the following embedding theorem [28].

Lemma 5.2. Suppose $T>\tau>0$. Then the following results hold: 
(1) if $0<p<(n+2) / n$ and $p \leq q \leq(n+2) p /(n+2-2 p)$, then there exits $C>0$ depending on $T-\tau, q$, and $p$ such that $\|u\|_{q, \tau, T} \leq C\|u\|_{W_{p}^{2,1}\left(Q_{\tau, T}\right)}$ for any $u \in W_{p}^{2,1}\left(Q_{\tau, T}\right)$;

(2) if $p=(n+2) / n$ and $p \leq q$, then there exits $C>0$ depending on $T-\tau$, $q$, and $p$ such that $\|u\|_{q, \tau, T} \leq C\|u\|_{W_{p}^{2,1}\left(Q_{\tau, T}\right)}$ for any $u \in W_{p}^{2,1}\left(Q_{\tau, T}\right)$;

(3) if $p>(n+2) / n$, then there exits $C>0$ depending on $T-\tau$ and $p$ such that $\|u\|_{\infty, \tau, T} \leq$ $C\|u\|_{W_{p}^{2,1}\left(Q_{\tau, T}\right)}$ for any $u \in W_{p}^{2,1}\left(Q_{\tau, T}\right)$;

5.2. Proof of Theorem 2.1. Let $N \in \mathbb{Z}^{+}$. We introduce a cut off function $\beta_{N} \in C^{\infty}\left(\mathbb{R}^{3}\right)$ with $0 \leq \beta_{N} \leq 1$ satisfying

$$
\beta_{N}(S, E, I)= \begin{cases}1, & |S|+|E|+|I| \leq N \\ 0, & |S|+|E|+|I| \geq 2 N .\end{cases}
$$

We define

$$
f_{N}(x, S, E, I, R)=\beta_{N}(S, E, I) f(x, S, E, I, R),
$$

and consider the system

$$
\left\{\begin{aligned}
\frac{\partial S}{\partial t} & =-f_{N}(x, S, E, I, R), & & x \in \bar{\Omega}, t>0, \\
\frac{\partial E}{\partial t} & =d_{E} \Delta E+f_{N}(x, S, E, I, R)-\lambda E, & & x \in \Omega, t>0, \\
\frac{\partial I}{\partial t} & =d_{I} \Delta I+\lambda E-\gamma I, & & x \in \Omega, t>0, \\
\frac{\partial R}{\partial t} & =\gamma I, & & x \in \Omega, t>0, \\
\frac{\partial E}{\partial \eta} & =\frac{\partial I}{\partial \eta}=0, & & x \in \partial \Omega, t>0,
\end{aligned}\right.
$$

where $d_{E}, d_{I}, \lambda$ and $\gamma$ are positive constants, with initial conditions

$$
\begin{aligned}
S(x, 0) & =S_{0}(x), & & x \in \bar{\Omega}, \\
E(x, 0) & =E_{0}(x), & & x \in \Omega, \\
I(x, 0) & =I_{0}(x), & & x \in \Omega, \\
R(x, 0) & =R_{0}(x), & & x \in \bar{\Omega} .
\end{aligned}
$$

Our desired result follows from the following two lemmas. In particular, by Lemma 5.3, (5.4)-(5.5) has a unique nonnegative global classical solution. By Lemma 5.4, we can choose $N>M$ such that the solution of (5.4)-(5.5) solves (2.1)-(2.3).

Lemma 5.3. Suppose that (A1)-(A4) hold and there exists $\epsilon \in(0,1)$ such that $S_{0}, E_{0}, I_{0}, R_{0} \in$ $C^{\epsilon}(\bar{\Omega})$ are nonnegative. Then there exists a unique quadruple $(S, E, I, R)$ providing a global classical, component-wise nonnegative solution of (5.4)-(5.5) on $\bar{\Omega} \times[0, \infty)$.

Proof. Let

$$
F_{N}(x, S, E, I, R)=\left(\begin{array}{c}
-f_{N}(x, S, E, I, R) \\
f_{N}(x, S, E, I, R)-\lambda E \\
\lambda E-\gamma I \\
\gamma I
\end{array}\right) .
$$

Then $F_{N}$ is Lipschitz continuous in $(S, E, I, R) \in\left(L_{p}(\Omega)\right)^{4}$ uniformly in $x \in C(\bar{\Omega})$ for any $p \geq 1$. Then using the contraction mapping theorem [36, (5.4)-(5.5) has a unique global solution

$$
(S(\cdot, t), E(\cdot, t), I(\cdot, t), R(\cdot, t)) \in C\left([0, \infty) ;\left(L_{p}(\Omega)\right)^{4}\right) \cap C\left((0, \infty) ; L_{p}(\Omega) \times\left(W_{2, p}(\Omega)\right)^{2} \times L_{p}(\Omega)\right)
$$

for any $p \geq 1$. In particular, $E(\cdot, t), I(\cdot, t) \in C\left([0, \infty), C^{\epsilon}(\bar{\Omega})\right)$. So, by the continuous dependence of ordinary differential equations on parameters $[9], S(\cdot, t), R(\cdot, t) \in C^{1}\left([0, \infty), C^{\epsilon}(\bar{\Omega})\right)$. This in turn 
implies $E, I \in C^{2,1}(\Omega \times(0, \infty))$, and therefore we have the existence of a unique classical global solutions of 5.4 - -5.5 .

Since

$$
\begin{gathered}
-\left.f_{N}(x, S, E, I)\right|_{S=0} \geq 0, \\
f_{N}(x, S, E, I)-\left.\lambda E\right|_{E=0} \geq 0, \\
\lambda E-\left.\gamma I\right|_{I=0} \geq 0, \\
\left.\gamma I\right|_{R=0} \geq 0,
\end{gathered}
$$

for all $(x, S, E, I, R) \in \bar{\Omega} \times[0, \infty)^{4}$, the rectangle $[0, \infty)^{4}$ is invariant for (5.4) -(5.5). By the invariant rectangle argument, the solutions of (5.4)-(5.5) are nonnegative 43.

Lemma 5.4. Suppose that (A1)-(A4) hold and there exists $\epsilon \in(0,1)$ such that $S_{0}, E_{0}, I_{0}, R_{0} \in C^{\epsilon}(\bar{\Omega})$ are nonnegative. Then there exists $M>0$ independent of $N$ such that the solution $(S, E, I, R)$ of (5.4)-5.5) satisfies

$$
0 \leq S(x, t), E(x, t), I(x, t) \leq M \quad \text { for all } \quad x \in \bar{\Omega}, t>0 .
$$

Proof. Since $\partial_{t} S \leq 0$, we have $S(x, t) \leq S_{0}(x)$ for all $x \in \bar{\Omega}$ and $t \geq 0$. Hence,

$$
0 \leq S(x, t) \leq\left\|S_{0}\right\|_{\infty, \Omega}, x \in \bar{\Omega}, t \geq 0 .
$$

Summing the first four equations of $(5.4)$ and integrating over $\Omega \times(0, t)$, we have

$$
\int_{\Omega}(S(x, t)+E(x, t)+I(x, t)+R(x, t)) d x=\int_{\Omega}\left(S_{0}(x)+E_{0}(x)+I_{0}(x)+R_{0}(x)\right) d x .
$$

Then we use a duality argument [25, 34, 35] to show the boundedness of $E$ in $L_{p}(\Omega \times(\tau, \tau+1))$ independent of $\tau \geq 0$ for all $1<p<\infty$. For given $\tau \geq 0$, we define $\psi_{\tau}(t)$ by

$$
\psi_{\tau}(t)= \begin{cases}0, & \text { if } t<\tau \\ t-\tau, & \text { if } \tau \leq t \leq \tau+1, \\ 1, & \text { if } t>\tau+1\end{cases}
$$

Let $\tilde{S}(x, t)=\psi_{\tau}(t) S(x, t), \tilde{E}(x, t)=\psi_{\tau}(t) E(x, t)$ and $\tilde{I}(x, t)=\psi_{\tau}(t) I(x, t)$. Then $(\tilde{S}, \tilde{E}, \tilde{I})$ solve the following:

$$
\left\{\begin{aligned}
\frac{\partial \tilde{S}}{\partial t} & =-\psi_{\tau}(t) f_{N}(x, S, E, I, R)+\psi_{\tau}^{\prime}(t) S, & & x \in \bar{\Omega}, t \in(\tau, \tau+2), \\
\frac{\partial \tilde{E}}{\partial t} & =d_{E} \Delta \tilde{E}+\psi_{\tau}(t) f_{N}(x, S, E, I, R)-\lambda \tilde{E}+\psi_{\tau}^{\prime}(t) E, & & x \in \Omega, t \in(\tau, \tau+2), \\
\frac{\partial \tilde{I}}{\partial t} & =d_{I} \Delta \tilde{I}+\lambda \tilde{E}-\gamma \tilde{I}+\psi_{\tau}^{\prime}(t) I, & & x \in \Omega, t \in(\tau, \tau+2), \\
\frac{\partial \widetilde{E}}{\partial n} & =\frac{\partial \tilde{I}}{\partial n}=0, & & x \in \partial \Omega, t \in(\tau, \tau+2),
\end{aligned}\right.
$$

and

$$
\begin{aligned}
& \tilde{S}(x, \tau)=0, \quad x \in \bar{\Omega}, \\
& \tilde{E}(x, \tau)=\tilde{I}(x, \tau)=0, \quad x \in \Omega .
\end{aligned}
$$

Let $p^{\prime}=p /(p-1)$ such that $1 / p+1 / p^{\prime}=1$. Let $\theta \in L_{p^{\prime}}(\Omega \times(\tau, \tau+2))$ with $\|\theta\|_{p^{\prime}, \Omega \times(\tau, \tau+2)}=1$ and $\theta \geq 0$. Let $\phi$ be the unique solution of (5.1) with $T=\tau+2$ and $d=d_{E}$. By (5.2), we have $\phi \geq 0$. By Lemma 5.1. there exists $C_{p^{\prime}}>0$ independent of $\theta$ such that

$$
\|\phi\|_{p^{\prime}, \Omega \times(\tau, \tau+2)},\|\Delta \phi\|_{p^{\prime}, \Omega \times(\tau, \tau+2)} \leq C_{p^{\prime}} .
$$


Multiplying the first two equations of 5.6 by $\phi$, adding up them, and integrating over $\Omega \times(\tau, \tau+2)$, we obtain

$$
\int_{\tau}^{\tau+2} \int_{\Omega}(\tilde{S}+\tilde{E}) \theta d x d t \leq-d_{E} \int_{\tau}^{\tau+2} \int_{\Omega} \tilde{S} \Delta \phi d x d t+\int_{\tau}^{\tau+2} \int_{\Omega} \phi \psi_{\tau}^{\prime}(S+E) d x d t .
$$

By 5.8 and Hölder's inequality,

$$
\int_{\tau}^{\tau+2} \int_{\Omega} \tilde{S} \Delta \phi d x d t \leq\left\|S_{0}\right\|_{\infty, \Omega} \int_{\tau}^{\tau+2} \int_{\Omega}|\Delta \phi| d x d t \leq\left\|S_{0}\right\|_{\infty, \Omega}(2|\Omega|)^{\frac{1}{p}} C_{p^{\prime}} .
$$

By the definition of $\psi_{\tau}$ and $(5.8)$,

$$
\int_{\tau}^{\tau+2} \int_{\Omega} \phi \psi_{\tau}^{\prime} S d x d t=\int_{\tau}^{\tau+1} \int_{\Omega} \phi S d x d t \leq\left\|S_{0}\right\|_{\infty, \Omega}|\Omega|^{\frac{1}{p}} C_{p^{\prime}}
$$

Therefore, by (5.9), we have

$$
\int_{\tau+1}^{\tau+2} \int_{\Omega} E \theta d x d t \leq\left(d_{E}+1\right)(2|\Omega|)^{\frac{1}{p}} C_{p^{\prime}}\left\|S_{0}\right\|_{\infty, \Omega}+\int_{\tau}^{\tau+1} \int_{\Omega} \phi E d x d t .
$$

We will use 5.10 to obtain the boundedness of $\|E\|_{p, \Omega \times(\tau, \tau+1)}$. We note that either

$$
\|E\|_{p, \Omega \times(\tau+1, \tau+2)} \leq\|E\|_{p, \Omega \times(\tau, \tau+1)}
$$

or

$$
\|E\|_{p, \Omega \times(\tau+1, \tau+2)}>\|E\|_{p, \Omega \times(\tau, \tau+1)}
$$

holds for each $\tau>0$.

Suppose that 5.12 holds. By Hölder's inequality, we have

$$
\begin{aligned}
\int_{\tau}^{\tau+1} \int_{\Omega} \phi E d x d t & =\int_{\tau}^{\tau+1} \int_{\Omega} \phi E^{\frac{2}{n+2}} E^{\frac{n}{n+2}} d x d t \\
& \leq\left(\int_{\tau}^{\tau+1} \int_{\Omega} \phi^{p^{\prime}} E^{\frac{2 p^{\prime}}{n+2}} d x d t\right)^{\frac{1}{p^{\prime}}}\left(\int_{\tau}^{\tau+1} \int_{\Omega} E^{\frac{n p}{n+2}} d x d t\right)^{\frac{1}{p}} \\
& \leq|\Omega|^{\frac{2}{(n+2) p}}\left(\int_{\tau}^{\tau+1} \int_{\Omega} \phi^{p^{\prime}} E^{\frac{2 p^{\prime}}{n+2}} d x d t\right)^{\frac{1}{p^{\prime}}}\|E\|_{p, \Omega \times(\tau, \tau+1)}^{\frac{n}{n+2}} .
\end{aligned}
$$

We further assume that $p>(n+2) / n$. Then $1<p^{\prime}<(n+2) / 2$. Let

$$
q=\frac{(n+2) p^{\prime}}{(n+2)-2 p^{\prime}} .
$$

By Lemmas 5.1 5.2 there exists $K_{q}>0$ independent of $\tau$ such that

$$
\|\phi\|_{q, \Omega \times(\tau, \tau+1)} \leq K_{q}\|\phi\|_{p^{\prime}, \Omega \times(\tau, \tau+1)}^{(2,1)} \leq K_{q} C_{p^{\prime}} .
$$

Notice that

$$
\begin{gathered}
\frac{q}{p^{\prime}}=\frac{(n+2)}{(n+2)-2 p^{\prime}}>1, \\
\frac{2 p^{\prime}}{n+2} \frac{q}{q-p^{\prime}}=1,
\end{gathered}
$$

and

$$
\frac{1}{p^{\prime}} \frac{q-p^{\prime}}{q}=\frac{2}{n+2}
$$


Therefore, by Hölder's inequality and (5.14), we have

$$
\begin{aligned}
\left(\int_{\tau}^{\tau+1} \int_{\Omega} \phi^{p^{\prime}} E^{\frac{2 p^{\prime}}{n+2}} d x d t\right)^{\frac{1}{p^{\prime}}} & \leq\left(\int_{\tau}^{\tau+1} \int_{\Omega} \phi^{p^{\prime} \frac{q}{p^{\prime}}} d x d t\right)^{\frac{1}{p^{\frac{p}{p}}}}\left(\int_{\tau}^{\tau+1} \int_{\Omega} E^{\frac{2 p^{\prime}}{n+2} \frac{q}{q-p^{\prime}}} d x d t\right)^{\frac{1}{p^{\frac{q}{p}} \frac{p^{\prime}}{q}}} \\
& =\|\phi\|_{q, \Omega \times(\tau, \tau+1)}\|E\|_{1, \Omega \times(\tau, \tau+1)}^{\frac{2}{n+2}} \\
& \leq K_{q} C_{p^{\prime}}\left\|S_{0}+E_{0}\right\|_{1, \Omega}^{\frac{2}{n+2}} .
\end{aligned}
$$

By 5.10$)-5.15$ and duality, we obtain

$$
\|E\|_{p, \Omega \times(\tau+1, \tau+2)} \leq L_{1}+L_{2}\|E\|_{p, \Omega \times(\tau+1, \tau+2)}^{\frac{n}{n+2}},
$$

where $L_{1}, L_{2}>0$ are dependent on $p$ and $\left\|S_{0}+E_{0}\right\|_{1, \Omega}$, but independent of $N$ and $\tau \geq 0$. By (5.16), there exists $M_{p}>0$ independent of $N$ and $\tau$ such that

$$
\|E\|_{p, \Omega \times(\tau+1, \tau+2)} \leq M_{p} .
$$

Therefore, if 5.12 holds, then we have 5.17). Combining this with 5.11), we can conclude

$$
\|E\|_{p, \Omega \times(\tau+1, \tau+2)} \leq \max \left\{M_{p},\|E\|_{p, \Omega \times(0,1)}\right\},
$$

for every $\tau \geq 0$ and $p>(n+2) / n$.

It remains to produce a bound for $\|E\|_{p, \Omega \times(0,1)}$ independent of $N$. Let $\theta \in L_{p^{\prime}}(\Omega \times(0,1))$ with $\theta \geq 0$ and $\|\theta\|_{p^{\prime}, \Omega \times(0,1)}=1$. Let $\psi$ be the unique nonnegative solution of 5.1 with $\tau=0, T=1$ and $d=d_{E}$. Multiplying the first two equations in (5.4) by $\theta$, adding up them, and integrating on $\Omega \times(0,1)$, we obtain

$$
\int_{0}^{1} \int_{\Omega}(S+E) \theta d x d t \leq-d_{E} \int_{0}^{1} \int_{\Omega} S \Delta \psi d x d t+\int_{\Omega}\left(S_{0}+E_{0}\right) \psi(x, 0) d x .
$$

Integrating the first equation of 5.1 on $\Omega \times(0,1)$, we have

$$
\begin{aligned}
\int_{\Omega} \psi(x, 0) d x & =\int_{\Omega} \psi(x, 1) d x+\int_{0}^{1} \int_{\Omega} \theta(x, t) d x d t \\
& \leq 0+|\Omega|^{\frac{1}{p}}\|\theta\|_{p^{\prime}, \Omega \times(0,1)}=|\Omega|^{\frac{1}{p}} .
\end{aligned}
$$

By $5.19-5.20$ and duality, we have

$$
\|E\|_{p, \Omega \times(0,1)} \leq\left(d_{E} C_{p^{\prime}}\left\|S_{0}\right\|_{\infty, \Omega}+\left\|S_{0}+E_{0}\right\|_{\infty, \Omega}\right)|\Omega|^{\frac{1}{p}} .
$$

By 5.18 and 5.21 , we have

$$
\|E\|_{p, \Omega \times(\tau, \tau+1)} \leq \max \left\{M_{p},\left(d_{E} C_{p^{\prime}}\left\|S_{0}\right\|_{\infty, \Omega}+\left\|S_{0}+E_{0}\right\|_{\infty, \Omega}\right)|\Omega|^{\frac{1}{p}}\right\}
$$

for $\tau \geq 0$ and $p>(n+2) / n$, where the right hand side of 5.22 is independent of $N$.

We can obtain a bound of $\|I\|_{p, \Omega \times(\tau, \tau+1)}$ independent of $N$ and $\tau \geq 0$ using a similar argument as above. Let $\theta \in L_{p^{\prime}}(\Omega \times(\tau, \tau+2))$ with $\|\theta\|_{p^{\prime}, \Omega \times(\tau, \tau+2)}=1$ and $\theta \geq 0$. Let $\bar{\phi}$ be the unique solution of (5.1) with $T=\tau+2$ and $d=d_{I}$. Multiplying the first three equations of 5.6) by $\bar{\phi}$, adding up them, and integrating over $\Omega \times(\tau, \tau+2)$, we obtain

$$
\begin{aligned}
\int_{\tau}^{\tau+2} \int_{\Omega}(\tilde{S}+\tilde{E}+\tilde{I}) \theta d x d t \leq & -d_{I} \int_{\tau}^{\tau+2} \int_{\Omega} \tilde{S} \Delta \bar{\phi} d x d t+\left(d_{E}-d_{I}\right) \int_{\tau}^{\tau+2} \int_{\Omega} \tilde{E} \Delta \bar{\phi} d x d t \\
& +\int_{\tau}^{\tau+2} \int_{\Omega} \bar{\phi} \psi_{\tau}^{\prime}(S+E+I) d x d t
\end{aligned}
$$


Using (5.22), we can bound the terms involving $\tilde{E}$ and $E$ in 5.23$)$. This will allow us to obtain an equality:

$$
\int_{\tau+1}^{\tau+2} \int_{\Omega} I \theta d x d t \leq C+\int_{\tau}^{\tau+1} \int_{\Omega} \phi I d x d t
$$

where $C$ depends on $p$ and $\left\|S_{0}\right\|_{\infty, \Omega}$ but independent of $N$ and $\tau$. This inequality is similar to (5.10) and can produce a bound of $\|I\|_{p, \Omega \times(\tau, \tau+1)}$ that is similar to 5.22 .

Finally, by Lemma 5.2 and the second equation of (5.6), there exists $C_{p}>0$ depending on $p$ independent of $N$ and $\tau$ such that

$$
\|E\|_{p, \Omega \times(\tau+1, \tau+2)}^{(2,1)} \leq\|\tilde{E}\|_{p, \Omega \times(\tau, \tau+2)}^{(2,1)} \leq C_{p}\left\|\psi_{\tau} f_{N}(\cdot, S, E, I, R)-\lambda \tilde{E}+\psi_{\tau}^{\prime} E\right\|_{p, \Omega \times(\tau . \tau+2)} .
$$

By (5.22) (a similar bound holds for $I$ ) and (A4), for each $p>0$ there exists $\bar{C}_{p}>0$ depending on $p$ independent of $N$ and $\tau$ such that

$$
\|E\|_{p, \Omega \times(\tau+1, \tau+2)}^{(2,1)} \leq \bar{C}_{p} .
$$

By Lemma 5.2, there exists $M>0$ depending on $p$ independent of $N$ and $\tau$ such that

$$
\|E\|_{\infty, \Omega \times(\tau+1, \tau+2)} \leq M .
$$

Using the third equation of (5.6), we can obtain a similar bound for $I$. This proves the lemma.

5.3. Proof of Theorem 2.2. Summing the first two equations of 2.1$)$ and integrating over $\Omega \times(0, t)$, we have

$$
\int_{\Omega}(S(x, t)+E(x, t)) d x+\lambda \int_{0}^{t} \int_{\Omega} E(x, s) d x d s=\int_{\Omega}\left(S_{0}(x)+E_{0}(x)\right) d x
$$

which implies $\|E\|_{1, \Omega \times(0, \infty)}<\infty$ and $\|E\|_{1, \Omega \times(\tau, \tau+1)} \rightarrow 0$ as $\tau \rightarrow \infty$. By (5.22) and Hölder's inequality, we have

$$
\|E\|_{q, \Omega \times(\tau, \tau+1)} \leq\|E\|_{1, \Omega \times(\tau, \tau+1)}^{\frac{1}{2}}\|E\|_{2 q-1, \Omega \times(\tau, \tau+1)}^{\frac{1}{2}} \rightarrow 0 \quad \text { as } \quad \tau \rightarrow \infty
$$

for all $1<q<\infty$. Similarly, we can sum the first three equations of 5.6 and integrating over $\Omega \times(0, t)$ to obtain

$$
\int_{\Omega}(S(x, t)+E(x, t)+I(x, t)) d x+\gamma \int_{0}^{t} \int_{\Omega} I(x, s) d x d s=\int_{\Omega}\left(S_{0}(x)+E_{0}(x)+I_{0}(x)\right) d x,
$$

which implies $\|I\|_{1, \Omega \times(0, \infty)}<\infty$ and $\|I\|_{1, \Omega \times(\tau, \tau+1)} \rightarrow 0$ as $\tau \rightarrow \infty$. By Hölder's inequality, we have $\|I\|_{q, \Omega \times(\tau, \tau+1)} \rightarrow 0$ as $\tau \rightarrow \infty$ for each $q>1$. Noticing (A4), for each $p>1$ we have

$$
\left\|f_{N}(\cdot, S, E, I, R)\right\|_{p, \Omega \times(\tau, \tau+1)} \rightarrow 0 \quad \text { as } \quad \tau \rightarrow \infty .
$$

It follows from 5.25 and Lemma 5.2 that $\|E(\cdot, t)\|_{\infty, \Omega} \rightarrow 0$ as $t \rightarrow \infty$. Similarly, we can prove $\|I(\cdot, t)\|_{\infty, \Omega} \rightarrow 0$ as $t \rightarrow \infty$.

Finally, we consider the asymptotic behavior of $S(x, t)$ as $t \rightarrow \infty$. Since $\partial_{t} S(x, t) \leq 0$ for all $x \in \bar{\Omega}$ and $t>0$, there exists a function $S_{\infty}(x) \geq 0$ such that $S(x, t) \rightarrow S_{\infty}(x)$ as $t \rightarrow \infty$ for each $x$. Multiplying the first equation of $(2.1)$ by $-1 / S(x, t)$, we obtain

$$
\begin{aligned}
\frac{d}{d t}-\ln (S(x, t)) & =\frac{f(x, S(x, t), E(x, t), I(x, t), R(x, t))}{S(x, t)} \\
& \leq \frac{g(S(x, t)) P(E(x, t), I(x, t))}{S(x, t)},
\end{aligned}
$$


where $g$ and $P$ are defined in (A4). Since $g$ is continuously differentiable, $g(0)=0$ and $S(x, t)$ is bounded, $g(S) / S$ is bounded. Since $P(E, I)$ is a polynomial, $P(0,0)=0$, and $E$ and $I$ are bounded, there exists $K>0$ such that

$$
\frac{g(S) P(E, I)}{S} \leq K(E+I)
$$

By this estimate and (5.27), we have

$$
\frac{d}{d t} \int_{O}-\ln (S(x, t)) d x \leq K \int_{O}(E(x, t)+I(x, t)) d x,
$$

where $O$ is any open subset of $\Omega$ on which $S_{0}>0$. Integrating (5.28) over $(0, \infty)$, we have

$$
-\int_{O} \ln \left(S_{\infty}(x)\right) d x<\infty
$$

Therefore, $S_{\infty}(x)>0$ a.e. in any open subset of $\Omega$ on which $S_{0}>0$.

\section{REFERENCES}

1. L. J. S. Allen, B. M. Bolker, Y. Lou, and A. L. Nevai, Asymptotic profiles of the steady states for an SIS epidemic reaction-diffusion model, Discrete \& Continuous Dynamical Systems 21 (2008), no. 1, 1-20.

2. Z. Bai, R. Peng, and X.-Q. Zhao, A reaction-diffusion malaria model with seasonality and incubation period, J. Math. Biol. 77 (2018), no. 1, 201-228. MR 3800806

3. G. Bertaglia, W. Boscheri, G. Dimarco, and L. Pareschi, Spatial spread of COVID-19 outbreak in Italy using multiscale kinetic transport equations with uncertainty, arXiv preprint arXiv:2106.07262 (2021).

4. Askery Canabarro, Elayne Tenório, Renato Martins, Lais Martins, S. Brito, and R. Chaves, Data-driven study of the COVID-19 pandemic via age-structured modelling and prediction of the health system failure in Brazil amid diverse intervention strategies, Plos one 15 (2020), no. 7, e0236310.

5. D. D. S. Candido, A. Watts, L. Abade, M. U. G. Kraemer, O. G. Pybus, J. Croda, W. De Oliveira, K. Khan, E. C. Sabino, and N. R. Faria, Routes for COVID-19 importation in Brazil, Journal of Travel Medicine 27 (2020), no. 3, taaa042.

6. F. Capone, V. De Cataldis, and R. De Luca, Influence of diffusion on the stability of equilibria in a reaction-diffusion system modeling cholera dynamic, Journal of mathematical biology 71 (2015), no. 5, 1107-1131.

7. C. Castilho, J. A. M. Gondim, M. Marchesin, and M. Sabeti, Assessing the efficiency of different control strategies for the COVID-19 epidemic, Electronic Journal of Differential Equations 2020 (2020), 1-17.

8. Center for International Earth Science Information Network - CIESIN - Columbia University. 2018. Gridded Population of the World, Version 4 (GPWv4): Population Density, Revision 11. Palisades, NY: NASA Socioeconomic Data and Applications center (SEDAC), https://www.who.int/publications/i/item/ responding-to-community-spread-of-covid-19, Accessed: 2021-10-6.

9. E. A. Coddington and N. Levinson, Theory of ordinary differential equations, Tata McGraw-Hill Education, 1955.

10. F. C. Coelho, R. M. Lana, O. G. Cruz, D. A. M. Villela, L. S. Bastos, Ana Pastore P., J. T. Davis, A. Vespignani, C. T. Codeço, and M. F. C. Gomes, Assessing the spread of COVID-19 in brazil: Mobility, morbidity and social vulnerability, PLoS One 15 (2020), no. 9, e0238214.

11. Coronavirus resource center, John Hopkins University \& Medcine, https://coronavirus.jhu.edu Accessed: 20219-1.

12. N. Crokidakis, Modeling the early evolution of the COVID-19 in Brazil: Results from a Susceptible-InfectiousQuarantined-Recovered (SIQR) model, International Journal of Modern Physics C 31 (2020), no. 10, 2050135.

13. R.-H. Cui, K.-Y. Lam, and Y. Lou, Dynamics and asymptotic profiles of steady states of an epidemic model in advective environments, J. Differential Equations 263 (2017), no. 4, 2343-2373. MR 3650341

14. L. R. P. de Alcantara, L. Silva, A. R. de Almeida, Maira G. da Rocha P., and A. P. Coutinho, Using different epidemiological models to modeling the epidemic dynamics in Brazil, medRxiv (2020).

15. G. B. de Almeida, T. Vilches, C. Ferreira, and C. M. C. B. Fortaleza, Several countries in one: a mathematical modeling analysis for COVID-19 in inner brazil, medRxiv (2020).

16. A. C. S. De Oliveira, L. H. M. Morita, E. B. Da Silva, L. A. R. Zardo, C. J. F. Fontes, and D. C. T. Granzotto, Bayesian modeling of COVID-19 cases with a correction to account for under-reported cases, Infectious Disease Modelling 5 (2020), 699-713. 
17. A. Eshragh, S. Alizamir, P. Howley, and E. Stojanovski, Modeling the dynamics of the COVID-19 population in Australia: A probabilistic analysis, Plos one 15 (2020), no. 10, e0240153.

18. W. E. Fitzgibbon, M. Langlais, and J. J. Morgan, A mathematical model of the spread of Feline Leukemia Virus (FeLV) through a highly heterogeneous spatial domain, SIAM journal on mathematical analysis 33 (2001), no. 3, $570-588$.

19. W. E. Fitzgibbon, J. J. Morgan, and G. F. Webb, An outbreak vector-host epidemic model with spatial structure: the 2015-2016 Zika outbreak in Rio De Janeiro, Theoretical Biology and Medical Modelling 14 (2017), no. 1, 1-17.

20. W. E. Fitzgibbon, J. J. Morgan, G. F. Webb, and Y. Wu, Analysis of a reaction-diffusion epidemic model with asymptomatic transmission, Journal of Biological Systems 28 (2020), no. 03, 561-587.

21. C. M. C. B. Fortaleza, R. B. Guimarães, G. B. de Almeida, M. Pronunciate, and C. P. Ferreira, Taking the inner route: spatial and demographic factors affecting vulnerability to COVID-19 among 604 cities from inner São Paulo State, Brazil, Epidemiology \& Infection 148 (2020), E118.

22. M. Gandhi, D. S. Yokoe, and D. V. Havlir, Asymptomatic Transmission, the Achilles Heel of Current Strategies to Control Covid-19, New England Journal of Medicine 382 (2020), no. 22, 2158-2160.

23. Github repository: covid19-monitorbr, https://github.com/endoedgar/covid19-monitorbr Accessed: 2021-10-6.

24. M. Grave, A. Viguerie, G. F. Barros, A. Reali, and A. L. G. A. Coutinho, Assessing the spatio-temporal spread of COVID-19 via compartmental models with diffusion in Italy, USA, and Brazil, arXiv preprint arXiv:2102.07208 (2021).

25. S. L. Hollis, R. H. Martin, Jr, and M. Pierre, Global existence and boundedness in reaction-diffusion systems, SIAM Journal on Mathematical Analysis 18 (1987), no. 3, 744-761.

26. C. Huang, Y. Wang, X. Li, L. Ren, J. Zhao, Y. Hu, L. Zhang, G. Fan, J. Xu, X. Gu, et al., Clinical features of patients infected with 2019 novel coronavirus in Wuhan, China, The lancet 395 (2020), no. 10223, 497-506.

27. A. Kapoor, X. Ben, L. Liu, B. Perozzi, M. Barnes, M. Blais, and S. O'Banion, Examining COVID-19 forecasting using spatio-temporal graph neural networks, arXiv preprint arXiv:2007.03113 (2020).

28. O. A. Ladyzhenskaia, V. A. Solonnikov, and N. N. Ural'tseva, Linear and quasi-linear equations of parabolic type, vol. 23, American Mathematical Soc., 1988.

29. H.-C. Li, R. Peng, and F.-B. Wang, Varying total population enhances disease persistence: qualitative analysis on a diffusive SIS epidemic model, J. Differential Equations 262 (2017), no. 2, 885-913. MR 3569410

30. Z. Liu, P. Magal, O. Seydi, and G. Webb, A COVID-19 epidemic model with latency period, Infectious Disease Modelling 5 (2020), 323-337.

31. __ Understanding unreported cases in the COVID-19 epidemic outbreak in Wuhan, China, and the importance of major public health interventions, Biology 9 (2020), no. 3, 50.

32. P. Magal, G. F. Webb, and Y. Wu, Spatial spread of epidemic diseases in geographical settings: Seasonal influenza epidemics in Puerto Rico, Discrete \& Continuous Dynamical Systems-B 25 (2020), no. 6, 2185-2202.

33. G. C. de Melo, R. A. de Araújo Neto, and K. C. G. M. de Araújo, Forecasting the rate of cumulative cases of COVID19 infection in Northeast Brazil: a Boltzmann function-based modeling study, Cadernos de Saúde Pública 36 (2020), e00105720.

34. J. J. Morgan, Global existence for semilinear parabolic systems, SIAM journal on mathematical analysis 20 (1989), no. $5,1128-1144$.

35. _ Boundedness and decay results for reaction-diffusion systems, SIAM Journal on Mathematical Analysis 21 (1990), no. 5, 1172-1189.

36. A. Pazy, Semigroups of linear operators and applications to partial differential equations, vol. 44, Springer Science \& Business Media, 2012.

37. R. Peng and X.-Q. Zhao, A reaction-diffusion SIS epidemic model in a time-periodic environment, Nonlinearity $\mathbf{2 5}$ (2012), no. 5, 1451-1471. MR 2914149

38. I. G. Pereira, J. M. Guerin, Andouglas G. Silva J., G. S. Garcia, P. Piscitelli, A. Miani, C. Distante, and L. M. G. Gonçalves, Forecasting Covid-19 dynamics in Brazil: a data driven approach, International Journal of Environmental Research and Public Health 17 (2020), no. 14, 5115.

39. M. F. do Prado, B. B. de P. Antunes, Leonardo dos Santos L. Bastos, Igor T. Peres, A. de A. B. da Silva, L. F. Dantas, F. A. Baião, P. Maçaira, S. Hamacher, and F. A. Bozza, Analysis of COVID-19 under-reporting in Brazil, Revista Brasileira de terapia intensiva 32 (2020), 224-228.

40. P. V. Savi, M. A. Savi, and B. Borges, A mathematical description of the dynamics of coronavirus disease 2019 (COVID-19): a case study of Brazil, Computational and Mathematical Methods in Medicine 2020 (2020).

41. R. A. Schulz, C. H. Coimbra-Araújo, and S. W. S. Costiche, COVID-19: A model for studying the evolution of contamination in Brazil, arXiv preprint arXiv:2003.13932 (2020). 
42. T. D. A. Serdan, L. N. Masi, R. Gorjao, T. C. Pithon-Curi, R. Curi, and S. M. Hirabara, COVID-19 in Brazil: Historical cases, disease milestones, and estimated outbreak peak, Travel medicine and infectious disease 38 (2020), 101733.

43. J. Smoller, Shock waves and reactiondiffusion equations, vol. 258, Springer Science \& Business Media, 2012.

44. G. J. B. Sousa, T. S. Garces, V. R. F. Cestari, T. Maria M. Moreira, R. S. Florêncio, and M. L. D. Pereira, Estimation and prediction of COVID-19 cases in Brazilian metropolises, Revista Latino-Americana de Enfermagem 28 (2020).

45. Y. Tang, T. D. A. Serdan, L. N. Masi, S. Tang, R. Gorjao, and S. M. Hirabara, Epidemiology of COVID-19 in Brazil: using a mathematical model to estimate the outbreak peak and temporal evolution, Emerging microbes \& infections 9 (2020), no. 1, 1453-1456.

46. Temporary Measures to Deter the Spread of COVID-19 in Brazil, https://www.mayerbrown.com/en/ perspectives-events/publications/2020/03/temporary-measures-to-deter-the-spread-of-covid-19-in-brazil Accessed: 2021-10-19.

47. G. L. Vasconcelos, A. M. S. Macêdo, R. Ospina, F. A. G. Almeida, G. C. Duarte-Filho, A. A. Brum, and I. C. L. Souza, Modelling fatality curves of COVID-19 and the effectiveness of intervention strategies, PeerJ 8 (2020), e9421.

48. A. Viguerie, A. Veneziani, G. Lorenzo, D. Baroli, N. Aretz-Nellesen, A. Patton, T. E. Yankeelov, A. Reali, T. J. R. Hughes, and F. Auricchio, Diffusion-reaction compartmental models formulated in a continuum mechanics framework: application to COVID-19, mathematical analysis, and numerical study, Computational Mechanics 66 (2020), no. 5, $1131-1152$.

49. W.-D. Wang and X.-Q. Zhao, Basic reproduction numbers for reaction-diffusion epidemic models, SIAM J. Appl. Dyn. Syst. 11 (2012), no. 4, 1652-1673. MR 3032845

50. X.-Y. Wang, X.-Q. Zhao, and J. Wang, A cholera epidemic model in a spatiotemporally heterogeneous environment, J. Math. Anal. Appl. 468 (2018), no. 2, 893-912. MR 3852557

51. What does community transmission of coronavirus mean?, https://www.abc.net.au/news/2020-04-11/ what-is-community-transmission-of-coronavirus-covid-19/12142638 Accessed: 2021-10-19.

52. World Health Organization: Responding to community spread of COVID-19, https://www.who.int/publications/ i/item/responding-to-community-spread-of-covid-19, Accessed: 2021-10-6.

53. Y.-X. Wu and X.-F. Zou, Asymptotic profiles of steady states for a diffusive SIS epidemic model with mass action infection mechanism, J. Differential Equations 261 (2016), no. 8, 4424-4447. MR 3537833

54. M. Yanes-Lane, N. Winters, F. Fregonese, M. Bastos, S. Perlman-Arrow, J. R. Campbell, and D. Menzies, Proportion of asymptomatic infection among covid-19 positive persons and their transmission potential: A systematic review and meta-analysis, PloS one 15 (2020), no. 11, e0241536.

Department of Mathematics, University of Houston, Houston, TX 77204, USA

E-mail address: wfitzgib@central.uh.edu

Department of Mathematics, University of Houston, Houston, TX 77204, USA

E-mail address: jjmorgan@central.uh.edu

Department of Mathematics, Vanderbilt University, Nashville, TN 37212, USA

E-mail address: glenn.f.webb@vanderbilt.edu

Corresponding author, Department of Mathematical Sciences, Middle Tennessee State University, MurfreesBORO, TN 37132, USA

E-mail address: yixiang.wu@mtsu.edu 